\title{
Soriginal Communirations.
}

\section{TEN YEARS OF OPERATIVE SURGERY IN THE PROVINCES.}

By Augustin Prichard, Esq., Surgeon, Clifton, Bristol.

\section{V.-Operations on the Eye. [Continued from page $8 \tilde{\text {. }}$ ]}

Cancer. CASE DCXI. J. A. W. aged $2 \frac{1}{2}$ years. In the right eye there had always been a white spot and it had been sightless and red and weak. A fortnight before I saw it the disease began to increase suddenly, and the cornea having sloughed the carcinomatous growth protruded from the eye. The gland in front of the ear became enlarged, I removed the contents of the orbit cleanly, and the child appeared quite well a week after, but a month afterwards the disease returned and shortly destroyed the patient.

The cancer-cells were well marked, and principally rounded.

CASE DCXII. J. J. aged $2 \frac{1}{2}$ years. This was a similar case, and I removed the contents of the orbit, about six months after the first appearance of the disease. It returned shortly and the child died about six months afterwards.

The former of these two cases was probably congenital, as cancer undoubtedly sometimes is, without any disease in either parent; and in the second it was of more recent growth. I have operated in other cases of a like nature, but I have no record of them; they were, however, all alike in one particular, viz., that the disease returned and the patients died.

Case DCxirr. L. C. aged 18. A strumous subject with threatening of disease of the lungs. The growth in the eye began as a tubercle on the iris, and I saw it from the commencement. After the lapse of a sear I extirpated the eye, and the patient had a tedious convalescence. She died about a year afterwards of tubercular phthisis, and there had been no return of the cancerous growth. The microscopic appearances were very characteristic, being cells, large, mostly round, with well marked nuclei and nucleoli.

CASE DCxiv. L. R., aged 31, presented himself first of all with a granular growth from the lower lid which was dissected away. Fifteen months after the first operation, the disease having returned, a second was performed, and actual cautery was applied to the surface. About eleven months afterwards (and thirty-two months after the first appearance of the disease), I removed the whole mass, with the eye and contents of the orbit. $\mathrm{He}$ died three months afterwards, nearly three years after the first onset of the complaint.

The microscopic examination of each portion of tumour was the same, namely nuclei and nucleoli, and round and fusiform cells. The cancerous growth had reached the brain.

CASE DCXv. W. B., aged 53, was the subject of a melanotic deposit underneath the conjunctiva, and close to the cornea, the tissues looking as if they had been stained. The disease gradually spread over the cornea, and to the conjunctiva of the lids, and I removed it a year after I first saw him. He was very much relieved for a time, but four months afterwards the tumour reappeared and grew rapidly, and he died after a period of excessive suffering and discomfort. $\mathrm{He}$ had melanosis of the brain, skin, peritoneum, and many of the other internal organs.

CaSe DCXvi. S. T., aged 47 , came under my care in consequence of internal mischief in the left eye which produced great dimness of sight and some pain, with a varicose condition of some of the conjunctival vessels. This state yielded to treatment, and she be- $\overrightarrow{\vec{F}}$ came relieved of her pain and discomfort, but the sight $\stackrel{\oplus}{+}$ was much impaired, and gradually lost. I saw her occasionally for a period of four years, during the greater $\frac{}{\sigma}$ part of which time no alteration took place, but $\overline{\bar{\omega}}$ subsequently the pain increased and became of $a \vec{\nabla}$ neuralgic character, and the interior part of the globe $\unrhd$ became more prominent, having exactly the appearance of the eye when it is affected with staphyloma corporis ${ }^{\infty}$ ciliaris. Her sufferings became so great, and the weak- $\overrightarrow{0}$ ness of the other eye interfered so much with her com-fort and occupation, that I advised her to submit to the $\vec{\omega}$ removal of the anterior part of the globe with the view $\sigma$ of allowing the humours to escape. I accordingly removed the cornea, but instead of the sudden gush of 3 clear humours which I had expected, I found the eyoi full of a black coagulum which I partially got rid of. $\omega$ This was in February of the year 1859, and by the fol. $\omega$ lowing April the disease had so far extended that another $\vec{\sigma}$ operation was requisite, and therefore I removed the globe and the contents of the orbit, and found the cavity full of black and tolerably firm cancerous tissue. There was free hæmorrhage for a short time, and for a few $\vec{V}$ weeks she was relieved. By October the tumour had reappeared, and extended forward nearly an inch from the orbit, reaching down to the level of the mouth, butc

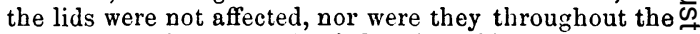
progress of the case. I tried various kinds of caustic, $\vec{\infty}$ namely, sulphuric acid, nitric acid, chloride of zinc, ni- $\infty$ trate of silver, caustic potash, and the dried sulphate of $\rightarrow$ zinc. The only one which seemed very effectual was a paste made with concentrated sulphuric acid and dried sulphate of zinc, and this answered admirably, but she could not bear its frequent application. Under its use the swelling was nearly destroyed and the lids becamed flaccid, but an attack of erysipelas interfered, and she음 was much weakened. There appeared to be no disposi- $\varrho$ tion in the disease to infect the surrounding tissues, nor $\overrightarrow{\vec{B}}$ was there any satisfactory evidence of disease in the $\frac{0}{3}$ other organs. At one time she had a great tendency whilst walking to fall forwards, and I thought that she had probably melanosis of the anterior lobes of the brain, but this symptom afterwards disappeared. She died rather suddenly about eighteen months after the first operation, and nearly six years after the onset of the disease.

In its earlier stages, this case might possibly have been benefited by an examination with the ophthalmoscope, which $I$ had never used at that time, although beyond the scientific interest of discovering a growth at the back of the eye it is more than probable that the resulto would have been the same. I can hardly think that the patient was the subject of this malignant disease for soN many years, and I think it more probable that it was an instance of secondary degeneration with a rapid development of the new cancerous tissue combined with black․ㅡ. pigment and a very vascular state. No post mortem ex-N amination was made.

Extirpation for the relief of the other Eye. CASECW Dcxvir. (Published before, Feb. 5, 1851.) J.F., agedo 46 , injured his left eye with a piece of iron thirteen years before I saw him, and he had frequently been laid upc with attacks of severe pain and inflammation, and for nine months previous to his becoming my patient he had been disabled. I removed the eye and on the tenth dayo he went out well, and returned to his work at onceThis was my first case of extirpation of the eye for thisd object.

CASE D(xvirI. T. B., aged 14, cut his left cornea, iris and lens with a butcher's knife, and ten weeks after the accident $I$ removed the remains of the eye (which was becoming atrophied), in consequence of dimness and in $\Omega$ tolerance of light in the other eje. He was quite curefo in four weeks. 
CAse nCxr. D. N., aged about 55 , had been under my care for many years, for an intense neuralgic condition of the face and head, apparently originating in an injured eye. The other eye was excessively weak, and he was quite unable to work. He had undergone all linds of treatment at my hands and under the care of other surgeons, and among the means used may be reckoned: blisters, leeches, mercury, setons, iron, quinine, opium, and ultimately, the long issue in the scalp, which latter relieved him much. I gave him some chloroforn, with the intention of removing his eye while he was insensible, but his pulse became so intermittent that I was obliged to operate without. He struggrled a good deal, and it was not an easy case, as the aperture of his eyelids was very small. He recovered and was able to return to his work and he grew stouter, but he had now and then an attack of neuralgic pain, although not comparable in severity to his former sufferings.

CaSE DCxx. C. H. aged 15. The right eye, which had been injured by a piece of crockery, and had given rise to much pain and intolerance of light and dimness of sight in the other eye, was removed by me in the usual way, under chloroform. She went out cured in a very short time.

CASE DCXXI. S. C. aged about 56. The right eye was removed in consequence of long standing disease involving the cornea and iris. I found considerable ditficulty in this case also, in consequence of the small aperture between the lids. The patient did remarkably well.

CASE DCXxrr. H.T. aged 55. This patient was nearly blind from internal disorganisation of the right eye. Her left eye was almost useless to her from the dimness and intolerance of light, and the disease in both had been of long standing. She refused to take chloroform, and therefore I operated without. She recovered well, and has retained the use of the other eye, which was rapidly failing before the operation.

The great peculiarity in this case was the condition of the eye. The cornea was opaque, rough, and yellow; the lens hard and also opaque was loose in the anterior chamber; the pigment internally had disappeared, and occupying the position of the choroid was a thin delicate shell of bone, brittle and hard and semi-transparent in its nature.

This ossification of the choroid coat has not been no. ticed much by authors, obviously on account of the want of opportunity of examining many specimens which existed before this operation became established. Gross, in his very comprehensive volume on pathological anatomy, gives an account of this state, but with that exception I have seen no notice of it.

The following case, the last I have to narrate of ex tirpation of the eye, is another instance of this nature.

CASE DCXXmr. G. H., aged 35, lost his left eye by an injury with a peg-top, when a young boy, and a few years before I saw him he had undergone an operation for the removal of the opaque lens in this eye. The operation did not succeed and constant pain and irritation with great intolerance of light followed it, and he was unable to attend to business. I removed the remains of the left eye, and he was better the next day than he had been for years, and the result of the case was very satisfactory.

There was partial collapse of the globe, and a folding of the sclerotic. The posterior part of the choroid was firmly ossified, with a small round hole in it, where the optic nerve was connected with the retina. The bony substance which replaced the choroid was thicker and firmer than in the former case, where it formed a delicate shell.

REMARKS. The instruments required for this operation are a curved scissors, a scalpel, a forceps, a strabismus hook, and the wire speculum. The globe only is to be removed, the muscles being divided close to the sclerotic, and no section of the outer commissure of the lids, re quired for the removal of cancerous and other tumours of the orbit, is necessary. The case is clearly one winere chloroform is advisable, although one of my patients (Case 630) preferred to submit without. After touching with a sharp scalpel round the conjunctiva, about the eighth of an inch from the edge of the cornea, I have found it convenient to raise the recti muscles one after the other upon a strabismus hook and divide thern, when the globe starts forward and the division of the optic nerve and other posterior connections is readily accomplished.

\section{CASE OF STRANGULATED HERNIA TREATED SUCCESSFULLY BY THE INVERTING METHOD.}

By G. Pound, Esq., Odiham.

Mrs. E., aged 50, has been the subject of hernia (oblique inguiual) of the right side for many years. She has also prolapsus uteri. She has at different times suffered very much with severe symptoms, as sickness and pain; and the hernia bas often been irreducible by her own efforts for some days. These attacks have lately become more frequent. She first became a patient of mine in March last. At that period she had sickness, constipation, pain in the abdomen, and the hernial tumour was as large as an orange, not very tender, but could not be returned. Under the use of opium and an enema, the symptoms subsided, and she was able to effect reduction herself. A second attack occurred in May; the same treatment was pursued with a like successful result.

I was called to the case for the third time on Monday the 22 rid July. I saw my patient at 6 P.s. She stated that, feeling poorly the past week, she had taken two "antibilious" pills; the last pill she had taken on the previous Saturday night, and her bowels were moved on Sunday morning. She had been very sick; and the hernial protrusion had occurred during violent retching, and had existed since midday on Sunday. The sicliness was, when I saw her, very troublesome; the countenance was anxious; the belly rather full and distended. The taxis failed to make any impression on the hernia. The tongue was clean; pulse 108 , weak. A pill con. taining opium was ordered to be taken every four hours, with two minims of hydrocyanic acid in mixture, and an injection to be administered immediately.

July 23, 10 A.n. The sickness was the same. The injection was returned immediately last evening. There was no action of the bowels. 'The tumour in the groin was in the same state. The opium and mixture were continued; and the enema was repeated; pulse 96.

6 P.sr. The husband came to my surgery, and informed me that the injection had acted; that she had not been sick since my visit in the morning; that the hernia was smaller; and that she had had a little sleep. My experience of the case led me to hope that the result would be as fortunate as on former occasions.

July 24,11 A.M. I found that the account given last evening by the husband of the patient was too fuvourably coloured. The sickness had now been very trouble. some since 3 A.nr. A little gruel tinged with fæcal matter was shown to me as the stool. The tumour had diminished nothing in size, and was a little ter,der on handling. About the neck there was considerable thickening. The tongue remained clean; pulse 96 . 'The countenance was somewhat anxious; the belly felt distended, but was not very tender. The enema was repeated; and she was ordered to take a grain of opium directly and another grain an hour afterwards.

3 P.Ir. Considering the probability to be strong that 169 